\title{
DESAFIOS DO DIREITO À CIDADE ÀS VULNERABILIDADES NA PERSPECTIVA DO PLURALISMO JURÍDICO
}

\begin{abstract}
${ }^{1}$ Gabriela Fauth
${ }^{2}$ Rosângela Lunardelli Cavallazzi

\section{RESUMO}

Este trabalho aborda o diálogo entre diferentes áreas do conhecimento, devido à complexidade e os múltiplos conflitos envolvidos nos estudos de natureza jurídicourbanística. O desafio contemporâneo inclui enfrentar novas vulnerabilidades que influenciam na perspectiva jurídica. Revisitar os conceitos tradicionais do Direito é relevante para uma mudança substancial, a partir das práticas sociais instituintes, buscando a construção permanente do pluralismo jurídico. Pretendeu-se analisar o desafio do campo jurídico a partir destas vulnerabilidades, em que o direito à cidade é a categoria analítica que pode contribuir para uma visão pluralista.
\end{abstract}

Palavras-chave: Pluralismo jurídico, Direito à cidade, Vulnerabilidades, Cidade standard, Alternativas transformadoras

\section{RETOS DEL DERECHO A LA CIUDAD FRENTE LAS VULNERABILIDADES EN LA PERSPECTIVA DEL PLURALISMO JURÍDICO}

\section{RESUMEN}

Este trabajo aborda el diálogo entre diversas áreas del conocimiento, a razón de la complexidad y de los múltiples conflictos involucrados en los estudios de naturaleza jurídicourbanística. El desafío contemporáneo incluye el enfrentamiento de nuevas vulnerabilidades que influyen en la perspectiva jurídica. Revisitar los conceptos tradicionales del Derecho es relevante para una mudanza substancial, a partir de las prácticas sociales instituyentes, buscando la permanente construcción del pluralismo jurídico. Se aspiró analizar el desafío del campo jurídico a partir de estas vulnerabilidades, en que el derecho a la ciudad es la categoría analítica que puede contribuir para una visión pluralista.

Palabras-claves: Pluralismo jurídico, Derecho a la ciudad, Vulnerabilidades, Ciudad standard, Alternativas transformadoras

\footnotetext{
1 Doutora em Direito Público pela Universitat Rovira i Virgili, Tarragona (Espanha). Bolsista de pós-doutorado da Fundação de Amparo à Pesquisa do Estado do Rio de Janeiro - FAPERJ, Rio de Janeiro. (Brasil). E-mail: gabrielafauth@hotmail.com

${ }^{2}$ Doutora em Direito pela Universidade Federal do Rio de Janeiro - UFRJ, Rio de Janeiro (Brasil). Pesquisadora da Universidade Federal do Rio de Janeiro, Rio de Janeiro (Brasil). E-mail: rosangela.cavallazzi@ gmail.com
} 


\section{INTRODUCCIÓN}

El presente artículo propone un análisis en el espacio de reflexión de la ciudad standard ${ }^{1}$, originaria de nuevas vulnerabilidades y consecuentes blindajes al derecho a la ciudad, se concretizando en la producción de su propio espacio. Es decir, cómo los procesos de estandarización se explicitan en las construcciones normativas? Estos desafíos exigen la promoción del diálogo entre campos disciplinares distintos, permitiendo además de la articulación de distintos aportes teóricos, principalmente una visión plural en el sentido de la comprensión de los conflictos urbanos, de escala local y regional. En el campo del Derecho, las estrategias de solución buscan superar la matriz moderna que administra demandas individuales y singulares. En esta perspectiva, un sistema cerrado, abstracto y pretensamente amplio sobre todas las situaciones no respeta las demandas de la sociedad contemporánea, llena de intereses y valores múltiples y plurales. En el actual proceso de globalización, el tiempo está aprisionado, viabilizando ciudades de mismo padrón, a ejemplo de los contractos de adhesión estandarizados, en que el espacio y las relaciones sociales urbanas están inmovilizados dentro del modelo de mercado.

En resumen, con la crisis de confianza (relaciones jurídicas, instituciones y políticas públicas) y, de forma más puntual, con la observación cotidiana de las insuficiencias del instrumental lógico-formal en el sentido sentido de comprender los conflcitos inherentes a la sociedad de consumo, el Derecho, muy lejos de solucionarlos, tampoco os ecuaciona. El hecho, sin duda, no constituye una novedad, ya que la matriz moderna (adoptada tanto en el campo jurídico como en el campo del urbanismo, por ejemplo) siempre ha sido muy competente en la producción de falsas igualdades.

Muchos caminos han sido trazados en el sentido de asumir y enfrentar los retos de la Modernidad (HOBSBAWN , 2007). Así siendo, el debate sobre la crisis de los paradigmas modernos (SOUZA JUNIOR, 2008) ${ }^{2}$ sigue manifestándose de forma explícita y contundente en el campo jurídico.

\footnotetext{
${ }^{1}$ Conceito construído a partir de pesquisas realizadas pelas autoras sobre cidades contemporâneas, especialmente a partir do entendimento de cidades que evidenciam a produção de novas vulnerabilidades e consequentes blindagens ao direito à cidade. CAVALLAZZI, Rosângela, Relatório de Pesquisa do projeto "Códigos da cidade: Análise das interferências jurídico-urbanísticas na cidade standard”. Rio de Janeiro: PROURB/UFRJ, 2012.

${ }^{2}$ Conforme José Geraldo de Sousa Júnior, “no final dos anos 1960, a crise de paradigmas de conhecimento e de ação humanas projetadas no mundo abriu, no campo jurídico, o mesmo debate crítico que se travava nos demais âmbitos sociais e teóricos. Sob o enfoque da crítica, portanto, e ao impulso de uma conjuntura política complexa em sua adversidade, notadamente no contexto social da realidade latino-americana, o pensamento jurídico ocidental buscou reorientar-se paradigmaticamente, rejeitando a matriz positivista de redução da complexidade ao formalismo legalista e
} 
A partir de una perspectiva paisajística, por ejemplo, se puede observar que el tipo de urbanización establecido en los días de hoy es bastante semejante, para no decir igual, y repetido en diferentes lugares, uniformizando también las relaciones sociales. En este aspecto, la ciudad standard, a su vez, amplía la vulnerabilidad agravada, materializándose en la producción del espacio. Y así, el habitante acaba por adherir a una ciudad que no permite diálogo, y su supervivencia cada vez más se constituye en espacios de desigualdad ${ }^{3}$.

Por tanto, el desafío del pluralismo jurídico en el compromiso con las prácticas sociales instituyentes cobra sentido al resolver conflictos de carácter global en las ciudades estándares, identificando metodologías y desarrollando alternativas de proyectos urbanísticos. A partir del análisis de las construcciones normativas en proyectos urbanísticos (TSIOMIS, 2006) la comprensión de los retos de la ciudad standard y el agravamiento de las vulnerabilidades resultan más claros.

Para este propósito, el diálogo entre diversas áreas del conocimiento se vuelve imprescindible a razón de la complexidad y de los múltiples conflictos que están involucrados en estudios de esta naturaleza. Las vulnerabilidades en relación a los derechos sociales que constituyen el derecho a la ciudad logran a la vez, relaciones, pero sobre todo, espacios, exigiendo, pues, planteamientos interdisciplinares.

El abordaje interdisciplinar en las investigaciones científicas ha permitido además del diálogo entre diversas áreas del conocimiento, principalmente, el acercamiento de conceptos y sus respectivas interpretaciones. En el sentido de la comprensión plural de las dinámicas sociales y, en relación al reconocimiento de la relevancia de las prácticas sociales.

El diálogo de saberes entre las diversas áreas del conocimiento podrá permitir que se deshaga mitos y sentidos comunes teóricos, identificando "viejos" paradigmas de la Modernidad, con la pretensión de ser suficiente para la identificación y "resolución" de los conflictos latentes o situados oriundos de las sociedades modernas y contemporáneas, explicitados por los movimientos sociales.

de deslocamento dos pressupostos éticos que fundam uma normatividade legítima”, p. 236. Ver más en SOUSA JÚNIOR (2008).

${ }^{3}$ Sobre este aspecto: no se puede revocar la ciudad. La afirmación, porun lado causa la seguridad de la permanencia, la certeza de la constancia de la producción de un paisaje producto de las relaciones sociales, por otro lado, causa , igualmente, la imposibilidad del cambio, el congelamiento de las formas y, además, la ineficacia de las normas y de su papel transformador. 
Esta interacción surge a partir de la contribución del derecho a la ciudad como compresión de las controversias urbanas, y como alternativa a los retos que urge la ciudad del siglo XXI, además de su sustancial vinculación con el cambio de paradigmas de la dogmática jurídica y la importancia de un examen entre teoría y práctica del Derecho. Afrontando desafíos, el derecho a la ciudad se materializa como un fenómeno del pluralismo jurídico y abre nuevos caminos para el análisis del Derecho Urbanístico contemporáneo (FAUTH, 2015).

Las constantes transformaciones del Derecho representan una visión del conocimiento que no es estática, sino que sigue en transición, bien como sucedió en su configuración y concepción anterior al proyecto desarrollado en la Modernidad. Conforme Gaston Bachelard, la experiencia científica es una experiencia que contradice la experiencia común. Por tanto, utilizando de la teoría concebida por él, existen obstáculos al pensamiento científico que merecen ser analizados y superados. Como es el caso del obstáculo que se centraba en un límite explicativo de un sistema jurídico inscrito en un paradigma, reduciendo la concepción del Derecho (BACHELARD, 1996).

Como es el caso del reconocimiento de nuevos centros normativos más allá del Derecho oficial, manifestado a partir del pluralismo jurídico.

El carácter experimental de este trabajo es justamente identificar el aporte del Derecho a este contexto señalado. El Derecho no es solo mediador de controversias, sino productor de espacio, de territorio, por ende, posee un papel fundamental también en la teoría de la producción del espacio (KONZEN, 2010).

Sin embargo, más que eso, la propuesta aquí planteada es que las diversas categorías pertenecientes al concepto del derecho a la ciudad puedan servir de base teórica a la búsqueda de la ciudad plural, democrática y justa, posibilitando la reducción de la vulnerabilidad social por medio del Derecho, del Urbanismo y de la política urbana. Para lograr esta finalidad la noción de eficacia social de la norma representa un método factible dentro del pluralismo jurídico (FARIA, 2004) ${ }^{4}$.

En este contexto, la relación entre Derecho, Urbanismo y otras áreas del saber, puede establecer una vía que permita contribuir para la eficacia social de la norma en la ciudad, fundamentado, pues, a partir del pluralismo jurídico.

Por este motivo, para Rosângela Cavallazzi (2007), el reconocimiento de los conflictos y de la fragmentación de la norma permite mucho más que identificar la debilidad de los derechos.

\footnotetext{
${ }^{4}$ José Eduardo Faria revela que "não se trata mais de pensar em uma racionalidade jurídica cujas categorias já não seriam definíveis a priori, mas tão só a posteriori...”. Ver en FARIA, José Eduardo, O Direito na Economia Globalizada, São Paulo, Malheiros, 2004, p. 274.
} 
Sino que admite un registro en el espacio simbólico del campo jurídico y, simultáneamente la cristalización de otra forma de espacio. Es decir, establece una competencia entre el Derecho codificado, homogeneizante de sentidos, de contenidos normativos con el pluralismo intrínseco de las prácticas sociales preestablecidas.

\section{NUEVOS SENTIDOS COMO INSTRUMENTO DE TRANSFORMACIÓN SOCIAL}

Entender que algunas áreas del Derecho han contribuido con el contexto de importantes cambios estructurales que caracterizan una transición sociohistórica -que muchos autores denominan crisis de la Modernidad e inicio de la Postmodernidad ${ }^{5}$, , rompiendo modelos políticos, históricos, sociales y culturales ${ }^{6}$, proporcionan la idea de que se introduzca igualmente una interpretación jurídica (WARAT, 1979), diferenciada de la hermenéutica jurídica.

De este modo, el debate sobre la crisis de los paradigmas modernos se manifiesta de forma contundente en el campo jurídico de forma más precisa a través de la observación cotidiana de las insuficiencias del instrumental lógico-formal para comprender los conflictos inherentes a la sociedad de consumo.

Cuestionar los orígenes, las permanencias y la búsqueda por el nuevo es desbloquear las ideas, es permitirse salir de pensamientos a lo mejor ideologizados y permitirse interpretar de otra manera el conocimiento (BACHELARD, 1996) ${ }^{7}$.

\section{LA TEORÍA JURÍDICA CRÍTICA DESDE EL ENFOQUE DEL PLURALISMO JURÍDICO}

Para entender la concepción pluralista del Derecho es necesario que nos reportemos a la

\footnotetext{
${ }^{5}$ Postmodernidad no es un concepto libremente aceptado y mucho menos unánime. Para algunos autores, inclusive, es un término equivocado. "Posmoderno probablemente no es un buen término, pues implica la idea de periodización histórica y periodizar es una idea todavía clásica o moderna", conforme LYOTARD (1992).

6 "Sempre houve uma tendência das elites nacionais a partilhar uma cultura "mundial" ou "global': Mas hoje estamos diante de algo bastante novo: um processo que leva as culturas a se adaptarem umas às outras numa escala planetária, com a difusão global da cultura popular de massa” en HOBSBAWN (2009).

7 "Segundo nossos adversários, segundo os filósofos, deveríamos considerar como fundamento da cultura científica as grandes generalidades. Como fundamento da mecânica: todos os corpos caem. Como fundamento da óptica: todos os raios luminosos se propagam em linha reta. Como fundamento da biologia: todos os seres vivos são mortais. Seriam assim colocadas, no limiar de cada ciência, grandes verdades primeiras, definições intocáveis que esclarecem toda doutrina. De fato, o início dos livros pré-científicos esbarra nesse esforço de definição preliminar, como é perceptível tanto na física do século XVIII como na sociologia do século XX. Pode-se, entretanto, questionar se essas grandes leis constituem pensamentos de fato científicos, ou, o que para nós dá no mesmo, pensamentos que sugerem outros pensamentos" (BACHELARD, 1996, p. 70).
} 
doctrina de la Institución. Esta doctrina constituye una reacción a la teoría estatal (CAVALLAZZI, 1993).

En la teoría estatal el Derecho se confunde con el Estado, consistiendo en una totalidad, un bloque homogéneo que excluye cualquier otro productor de juridicidad que no el propio Estado.

Los teóricos de la Institución ${ }^{8}$ intentan definir el Derecho como Institución y no como norma.

La teoría de la pluralidad de los ordenamientos jurídicos representada en la obra de Santi Romano ("L'ordinamento giuridico", 1918) marco pionero. Preocupado con la "crisis del Estado" (crisis do Estado italiano en principios del siglo XX), Romano hace una reflexión sobre los vínculos entre la crisis del Estado y la teoría del pluralismo jurídico (BOBBIO, 1980), enfatizando la existencia de grupos intermedios entre los individuos y el Estado (CAVALLAZZI, 1993).

Conforme CARBONNIER (1980), no existe un único pluralismo jurídico, sino diversos fenómenos de pluralismo jurídico.

LAJOIE (1998) en análisis de la obra de Jean Carbonnier afirma que la sociología jurídica debe proceder desde el punto de vista de que el Derecho es "esencialmente múltiple y heterogéneo". Para éste autor en un mismo momento y espacio social pueden coexistir varios sistemas jurídicos.

WOLKMER (2013), considera el pluralismo como una opción que no combate el Estado, y por eso es considerado emancipador, ya que más que nada es una forma de descentralizar el poder estatal y distribuirlo a toda una comunidad o a toda la sociedad que se ve identificada con alguna práctica.

La doctrina pluralista "no prevé la extinción del Estado, sino solamente la reducción de su esfera de actividad". Es decir, no determina la unidad del Derecho y posee un planteamiento propio para superar la crisis del Estado (BOBBIO, 1991).

El jurista George Gurvitch es uno de los precursores de la teoría del pluralismo. Y según BOBBIO (1980), es una de las formas más interesantes de crítica al Estado moderno visto en la contemporaneidad.

Uno de los dos movimientos críticos del Derecho nacido en la década de 1970 es la Asociación crítica del Derecho. Es un movimiento desarrollado en universidades francesas inspirado en Marx. Advertía para la necesidad de hacer una crítica al derecho positivo, una crítica profunda a las instituciones jurídicas, como el poder judiciario y, repensar la enseñanza del Derecho. Este movimiento repercute en Italia en la misma década, cuando magistrados italianos

\footnotetext{
${ }^{8}$ Dentre los teóricos de la Institución: Gurvitch, G.; Hauriou, M.; Santi Romano, entre otros
} 
rompen con la estructura judiciaria (por desilusión con los casos de corrupción y acción de la mafia) y pasan a entender que el jurista igualmente debe ser ciudadano, preocupado con las prácticas sociales, desarrollando la llamada Hermenéutica alternativa del Derecho. Eso es, una interpretación alternativa del Derecho que revisa y explota en profundidad los posibles vacíos del derecho positivo (FAUTH, 2010).

Siguiendo a WOLKMER (2013) de estos movimientos emerge el pluralismo tal cual se lo concibe hoy, llegando a América Latina en los años de 1980, primero en México a través de Oscar Correas (2007). La crítica de Latinoamérica se materializa a partir del nuevo constitucionalismo latinoamericano, por ser emancipadora, progresista y auténticamente periférica, contemplando nuevos sujetos emergentes ${ }^{9}$.

La gran meta del pluralismo es cuestionar el derecho positivo, el formalismo del Derecho, sus falacias y su compromiso con las élites. Por tanto, a través del pluralismo jurídico se reconoce la fuerza de los movimientos sociales. Y esto es un hecho muy importante, puesto que muchas de las prácticas sociales se materializan a través de los movimientos sociales (SOUSA JÚNIOR, 2008).

Por tanto no nos equivoquemos considerando que las prácticas sociales instituyentes sean una fuerza que anula la regla jurídica; justamente al revés, las prácticas sociales instituyentes constituyen la genuina fuerza social, el espacio de los vulnerables con sus prácticas autenticas, la frontera móvil del espacio de los posibles ${ }^{10}$ no campo jurídico.

Para empezar, cabe señalar WARAT (1990) que afirma que en el positivismo hay un abuso estatal del Derecho. Es decir, que el Estado excede cuando busca su lugar en la esfera normativa, puesto que provoca la alucinación en el otro y exalta un proyecto político-jurídico que se basa en sí mismo.

Sin embargo, los propios ordenamientos son también procesos históricos y sociales y que, por tanto, se actualizan continuamente (SOUSA JÚNIOR, 2010). Por esta razón, esta "actualización" se debe a la crisis del Estado moderno que acaba por abrazar el pluralismo jurídico como forma de crítica.

\footnotetext{
${ }^{9}$ Eso explica porque la mayor parte de la bibliografía sobre pluralismo jurídico en este trabajo sea de autores latinoamericanos.

Para WOLKMER (2013), autor del concepto de pluralismo jurídico emancipador, dos elementos son llaves en el pluralismo latinoamericano: a) construir una crítica a partir de la materialidad histórica de América Latina estructurales económicas y sociales - b) construir una crítica desde el nivel epistémico, a nivel de conocimiento, de las ideas, de las representaciones de mundo, de la cultura lationoamericana que permita la realización de una teoría, de un pensamiento que debe ser llevado a otras áreas, como la filosofía, la sociología hacia el mundo jurídico (uno de los más difíciles retos).

${ }^{10}$ Este concepto fue muy bien estudiado por Pierre Bourdieu (BOURDIEU, 1989, p. 211).
} 
El pluralismo ante todo es un modelo jurídico contrario y en contraposición al modelo oriundo de la Modernidad, centrado en el monismo jurídico.

En el pluralismo "el Estado no es más que uno de los posibles ordenamientos jurídicos, entre los cuales el hombre desarrolla su vida social, precisamente es el ordenamiento jurídico de la sociedad política" (SOUSA JÚNIOR, 2008).

En otras palabras, siguiendo este argumento, el Derecho entendido como un hecho social es la base del pluralismo jurídico.

En relación al concepto de derecho social, WOLKMER (2001) sostiene que "o Direito social tem sua fonte na coletividade organizada, na confiança e na participação de indivíduos que compõe grupos igualitários em colaboração", por ende lo mismo se materializa desde dentro. El mencionado jurista considera que la teoría desarrollada por Georges Gurvitch tiene un enorme valor para la sociología y filosofía jurídica.

Los diversos fenómenos de pluralismo jurídico han sido manifestados de formas diversas, como fenómeno colectivo o de grupo (CARBONNIER, 1980) ${ }^{11}$.

La crisis es de transición, ya que toda la sociedad se ve afectada por incertidumbres y por complejidades sociales que derivan en nuevas demandas y en el reconocimiento de nuevos conflictos.

En este contexto se interponen los desafíos, ya que, la creencia de que la teoría se adapta "dócilmente" a las relaciones sociales emergentes, a fin de atender el interés de todos, no encuentra respaldo en el proceso de interpretación flexible de la norma. En cambio, lo que ocurre es que hay una absorción indiscriminada de las prácticas emergentes en favor de un proceso de juridización de tendencias para justificar la eliminación de las excepciones en beneficio de la regularización y del reconocimiento de las prácticas sociales (CAVALLAZZI, 2007).

En este sentido, SOUSA SANTOS (2000) declara que eso sigue siendo evidente dato que la regulación social continúa hoy en día disponiendo de dos fortísimos factores de legitimación: la ciencia y el Derecho modernos. Tanto una como el otro reclaman para sí una eficacia y una coherencia que de hecho no tienen ni nunca tuvieron. Con todo, la consagración política y cultural de la que se han beneficiado en estos doscientos años provoca que, en la primera fase de transición

${ }^{11}$ CARBONNIER (1980) escribe sobre la manifestación del pluralismo asimismo como un fenómeno individual, calificado en Derecho y en Teologia como “objeción de consciencia”. “Objeción de consciencia (...) es la colisión de dos ordenes jurídicas en la consciencia individual”, p. 54. 
paradigmática, su legitimación se reproduzca independiente de la calidad de sus realizaciones prácticas.

Pese las resistencias de los legalistas y positivistas, es innegable que, paralelo y conjuntamente con el Derecho estatal, existan otros sistemas jurídicos que, igualmente, comprenden nuevas formas de conducta y, por supuesto, las reivindicaciones por nuevas formas jurídicas.

"En la transición paradigmática, la emancipación social es una aspiración tan obvia como inverosímil" (SOUSA SANTOS, 2000). Según el mencionado autor, es a partir de la utopía que el conocimiento emancipador consolidará su trayectoria epistemológica que va del colonialismo hacia la solidaridad. De este modo, será posible un nuevo perfil de coherencia y eficacia que tornará la emancipación en algo menos obvio y más verosímil.

Así, desde un punto de vista social, el Derecho, o el fenómeno jurídico puede entenderse partiendo de dos aspectos jurídicos entre sí. En primer lugar, el Derecho como discurso, esto es, como unidad de significación o práctica enunciativa en un contexto de producción; o, más precisamente, como discursos, en plural. Y en segundo lugar, el Derecho como espacio: sistema, conjunto de aparatos especializados que le sirven de soporte o, dicho de una forma más precisa en el lenguaje de BOURDIEU (2001), como campo social ${ }^{12}$ en el que se produce y negocia ese discurso.

El Derecho, desde esa perspectiva, no constituye sino el resultado de las luchas que se desarrollan entre agentes especializados, en competencia por el monopolio del capital jurídico, esto es, en concurrencia por el derecho a decir qué es lo que dice el Derecho. En realidad no se trata sino de una más de las fantasías sociales que, rodeadas de prestigio y misterio, esconden, bajo estrategias de universalización, las relaciones de dominación que se traducen en formas jurídicas (BOURDIEU, 2001). Conforme CAVALLAZZI (2007)

\begin{abstract}
"O grande desafio do direito instituído (para não perder a sua hegemonia) é estar em condições de "adotar" (absorver) ou neutralizar as manifestações normativas não-estatais, sem, contudo, descaracterizar-se enquanto forma jurídico/liberal, e ter a busca pela legitimidade como objetivo perpétuo, já que a legitimidade absoluta do regime político é uma utopia".
\end{abstract}

Resulta que no hay otra opción que sensibilizar tanto el jurista como el legislador. Si el sentido común teórico de los juristas, conforme afirma WARAT (1979), está en la mentalidad y en

\footnotetext{
12 "El campo social es una construcción analítica mediante la que designar un conjunto específico y sistemático de relaciones sociales; es decir, se trata de una especie de sistema, definible solo históricamente, que permite trasladar al análisis social la dinámica de relaciones que se desarrollan en la práctica”. Vid. BOURDIEU, 2001, p. 15.
} 
la práctica cotidiana de los operadores del Derecho (GONÇALVES, 2007) -, hay que realizar este cambio a través de nuevos abordajes jurídicos. Para este trabajo, esto se afirma a partir del Derecho urbanístico y su especial vínculo con el planeamiento urbanístico y su papel en la producción del espacio urbano.

El pluralismo jurídico, de este modo considerado, conserva importantes influencias sobre el ordenamiento de las ciudades. No es novedad que las circunstancias sociales y económicas repercutan en la configuración del espacio urbano. Tampoco se niega el hecho de que la configuración del espacio no prescinde de la utilización de instrumentos jurídicos de forma adecuada y actualizada.

Si la sociedad es cada vez más plural y si la idea de una ciudad más justa y democrática está caracterizada por la pluralidad, parece evidente que reconocer el pluralismo en todas sus formas será adecuado, por tanto, el pluralismo jurídico viene a corroborar este ideal de ciudad/sociedad y a través de sus mecanismos mediar los conflictos urbanos.

En este contexto de "explosión" del pluralismo en el capitalismo global y de la complejidad de la regulación jurídica. La transformación del Derecho refleja esa porosidad y ambigüedad del Derecho contemporáneo.

\section{EL DERECHO A LA CIUDAD COMO ALTERNATIVA PLURALISTA}

La tutela del derecho a la ciudad contempla un Derecho Urbanístico más allá del Derecho Administrativo, ya que su especificidad alcanza e incide de forma e intensidades distintas más que en el ámbito municipal ${ }^{13}$.

Con referencia a esta constatación, el derecho a la ciudad pasa a ser un presupuesto de la sostenibilidad, para el cumplimiento de las funciones sociales de la ciudad (DIDONET, 2010).

Como objetivo buscado por la política urbana, el derecho a la ciudad es la expresión de la dignidad de la persona en la producción del espacio, o, conforme Liana Portilho de Mattos: “[...] um compromisso com a dignidade da pessoa humana e com a garantia dos direitos humanos fundamentais: moradia, trabalho, saúde, educação, lazer, acesso à justiça, segurança, entre tantos outros" (MATTOS, 2001).

\footnotetext{
${ }^{13}$ La Carta Mundial por el derecho a la ciudad parte de tres ejes, reconociendo la función democrática de la ciudad, la función social de la propiedad y del suelo y, la participación ciudadana vinculante.
} 
Asimismo, comprendemos que el derecho a la ciudad como un derecho humano, núcleo de un sistema compuesto por un eje de derechos sociales, incluyendo el derecho a vivienda (implícito la regularización de la tierra), a educación, al empleo, a la salud, a los servicios públicos, al ocio, a la seguridad, al transporte público, a la información, a la preservación del patrimonio cultural, histórico y paisajístico, al medio ambiente natural y construido equilibrado (implícita la garantía del derecho a ciudades sostenibles). Según nuestra concepción no se establece cualquier jerarquía entre ellos (CAVALLAZZI, 2008; CAVALLAZZI, 2000).

No existe cualquier nivel de jerarquía entre los derechos sociales que componen el derecho a la ciudad. De hecho la efectividad del derecho a la ciudad está en la razón directa de la articulación que promueve el diálogo entre el Derecho Urbanístico y el Derecho Ambiental, en el sentido que se puede afirmar que la perspectiva jurídica urbano-ambiental es esencial para garantizar una ciudad sostenible.

Teniendo en cuenta que el derecho a la ciudad es un derecho humano que posiciona los individuos en el territorio, será a partir de esta distribución espacial que se ejercerán todos los derechos sociales que lo constituyen. Estos derechos sociales que se proyectan sobre el territorio, acompañados de las tutelas medioambiental y urbanística, están articulados de manera que componen el derecho fundamental a la calidad de vida (MARTÍN MATEO, 1988) ${ }^{14}$.

El derecho a la ciudad es mucho más que la libertad individual de acceder a los recursos urbanos: se trata del derecho a cambiarnos a nosotros mismos cambiando la ciudad. Es, además, un derecho común antes que individual, ya que esta transformación depende inevitablemente del ejercicio de un poder colectivo para remodelar los procesos de urbanización (HARVEY, 2008).

La fragmentación de la norma y de la forma, en el contexto de la globalización desmantela unidades y alcanza el ámbito económico y, especialmente los ámbitos político y social. Este proceso genera la determinación de espacio entre los riscos urbanos y fragiliza los derechos que componen el derecho a la ciudad. Este razonamiento está en el ambiente de la fragmentación, $a$ contracorriente sería el momento oportuno para reconocer nuevos derechos y prácticas sociales instituyentes.

\footnotetext{
${ }^{14}$ Con respecto a la calidad de vida ver MARTÍN MATEO, Ramón, "La calidad de vida como valor jurídico". Revista de Administración Pública, núm. 117, septiembre-diciembre 1988, pp. 51-70.

De acuerdo al autor, "la calidad de vida es en términos generales una noción intrínsecamente valorativa, que dependerá de los criterios, inevitablemente subjetivos, de quines ponderen una situación frente a otra (...)”, p. 56.

Reitera el mismo que en este concepto es imprescindible tener en cuenta los siguientes elementos: libertad, seguridad, trabajo, educación, nivel de vida, entorno físico, entorno social, salud y justicia. p. 58.
} 
Siguiendo las controversias y disputas de la ciudad y en la ciudad, la idea planteada aquí, no es solamente de que el derecho a la ciudad sea el instrumento de combate a vulnerabilidades, además de eso, debe ser usado por el planeamiento y las políticas urbanas como herramienta en la construcción de posibilidades a favor de una ciudad realmente plural.

Finalmente, el pluralismo permite la factibilidad del derecho a la ciudad. Sin embargo, se empieza por abordar algunas concepciones para evaluar el avance de la construcción teórica pluralista. Por este motivo el Derecho no deja de ser pieza importante en este escenario, a partir de la práctica del planeamiento, hacía la formación del espacio urbano como campo sociopolítico (FAUTH, 2015).

\section{CONSIDERACIONES FINALES}

A modo de conclusión, este ensayo pretendió expresar claramente cómo abrir espacio para que se repensara nuevas perspectivas jurídicas dentro de la dimensión normativa del planeamiento urbano, con la finalidad de mejor vislumbrar el proceso de construcción del espacio urbano en el contexto actual de ciudades fragmentadas y globalizadas. La producción de un discurso jurídico sensibilizado por la realidad y nutrido por otras áreas del conocimiento, como componentes esenciales de la sociedad y de la ciudad de este siglo permite tal espacio de reflexión.

Procediendo a analizar y percibir el fenómeno jurídico, aún siguiendo la crítica al modelo de ciudad y de planeamiento actuales, decurrentes de paradigmas con matriz lógico-formal se problematizó el saber jurídico dominante en la Modernidad y se reflexionó sobre las preocupaciones de la sociedad actual a partir del pensamiento crítico. Contexto en que el pluralismo posee papel fundamental.

En se tratando del espacio de las ciudades y con el propósito de contribuir con la dimensión jurídica en la construcción y formación del espacio urbano, resulta coherente contemplar los éxitos alcanzados, principalmente por el derecho a la ciudad, como nuevo paradigma jurídico.

La eficacia social de la norma urbanística, según los criterios de la incidencia, finalidad y legitimidad significando la incidencia continua de la legitimidad, por lo tanto, manifiesta la fiel relación de la plena efectividad de los derechos sociales que constituyen el derecho a la ciudad para construir políticas públicas afines con las demandas de este siglo, en correspondencia con las llamadas prácticas sociales instituyentes.

Así, dentro de la paradoja que es la Postmodernidad y que trajo nuevas realidades dentro del mundo globalizado, y al mismo tempo permite el crecimiento de alternativas para el mundo que se enfrenta hoy. La crisis es una alerta, una vez que el instrumental jurídico en su generalización 
sigue siendo aquel arraigado a la cultura pasada, por este motivo, las repuestas, en la mayoría de los casos, persigue priorizando prácticas desarticuladas con la realidad social, particularmente en los ámbitos urbano y ambiental.

Por consiguiente es viable aliar el derecho a la ciudad como una estrategia del pluralismo jurídico, competente y coherente con los enfrentamientos contemporáneos.

Aunque no sea una novedad referirse a un cambio de paradigmas, visto que es un debate que hace mucho ya se realiza, es importante verificar que el reconocido agravamiento de las vulnerabilidades en nuevas etapas del proceso de globalización permite también estrategias y la construcción de nuevos modelos en el plan del reto del derecho a la ciudad en la perspectiva del pluralismo jurídico.

\section{REFERENCIAS}

BACHELARD, G. A formação do espírito científico: contribuição para uma psicanálise do conhecimento, Tradução de Estela dos Santos Abreu, Rio de Janeiro: Contraponto, 1996.

BOBBIO, N. Contribución a la Teoria del Derecho. Valencia: Fernando Torres editor, 1980.

, El tiempo de los Derechos, Traducción Rafael de Asís Roig, Madrid: Editora Sistema, 1991.

BOURDIEU, P. Poder simbólico, Lisboa: Difel, 1989.

- Poder, Derecho y clases sociales, $2^{\mathrm{a}}$ ed., Traducción A. García Inda, Desclée de Bilbao: Brouwer editorial, 2001.

CARBONNIER, J. As Hipóteses Fundamentais da Sociologia Jurídica Teórica, In SOUTO, C. e FALCÃO, J. (orgs.) Sociologia do Direito, São Paulo: Pioneira, 1980.

CAVAllAZZI, R.L. O Plano da Plasticidade na Teoria Contratual. Rio de Janeiro. Tese (Doutoramento) - Universidade Federal do Rio de Janeiro. 1993.

- Relatório de Pesquisa do projeto "Códigos da cidade: Análise das interferências jurídico-urbanísticas na cidade standard”. Rio de Janeiro: PROURB/UFRJ, 2012. 
Tutela Constitucional do Direito à Cidade. $10^{\circ}$ Congresso Brasileiro de Direito Ambiental. Gestão de Riscos Ambientais: Licenciamento e outros Instrumentos. São Paulo, junho de 2005.

. O Estatuto epistemológico do direito urbanístico brasileiro. In: COUTINHO, R.; BONIZZATO, L. (Org.). Direito da Cidade: novas concepções sobre as relações jurídicas no espaço social urbano. Rio de Janeiro: Lumen Juris, 2007. p. 35-51.

Práticas sociais instituintes e sua tradução jurídica e urbanística. Projeto Integrado de Pesquisa Interinstitucional. RTDC - Revista trimestral de Direito Civil. Ano I, Vol. I JAN/MAR 2000.

. Novas Fronteiras do Direito Urbanístico. In TEPEDINO, G.FACHIN, L.E. (orgs.) O Direito e o tempo embates jurídicos e utopias contemporâneas - Estudos em homenagem ao Professor Ricardo Pereira Lira. Rio de Janeiro: Renovar, 2008. pp. 683-709.

CORREAS, O. (Coord.) Derecho indígena mexicano I. México, Coyoacán: UNAM-Ed. 2007.

DIDONET, N.A. Direito à Cidade sustentável: um conjunto de direitos humanos que se complementam. In CAVALLAZZI, R.L.; RIBEIRO, C.R. (Orgs.). Direito e Urbanismo: paisagem e direito à cidade. $1^{\text {a }}$ ed. Rio de Janeiro: PROURB - UFRJ, 2010, v. 1, p. 24

FARIA, J.E. O Direito na Economia Globalizada. São Paulo: Malheiros, 2004.

FAUTH, G., Crisis urbana y derecho a la ciudad: el espacio urbano litoral de Barcelona. Tesis de Doctorado, Universitat Rovira i Virgil, Tarragona, Espanha, 2015.

GONÇALVES, M.R.G. Surrealismo Jurídico: a invenção do Cabaret Macunaíma, Uma concepção emancipatória do Direito. Dissertação de mestrado, Universidade de Brasília, Faculdade de Direito, 2007.

HARVEY, D. El derecho a la ciudad, NLR, núm. 53, 2008, pp. 23-39, p. 23.

HOBSBAWN, E. Globalização, Democracia e Terrorismo. Trad. José Viegas. São Paulo: Companhia das Letras, 2007.

. O novo século: entrevista a Antonio Polito. São Paulo: Companhia das letras,

2009.

KONZEN, L.P. Espaços públicos urbanos e pluralismo jurídico: dos bens de uso comum do povo ao direito à cidade. Dissertação de Mestrado, Universidade Federal de Santa Catarina, 
Florianópolis, 2010.

LAJOIE, N.G. El pluralismo jurídico en Carbonnier, Anuario de filosofia del derecho, núm. 15, 1998.

LYOTARD, F. Qué es lo posmoderno? Zona Erógena, núm. 12, 1992.

MARTÍN MATEO, R. La calidad de vida como valor jurídico. Revista de Administración Pública, núm. 117, septiembre-diciembre 1988, pp. 51-70.

MATTOS, L.P. Arts. $1^{\circ}, 2^{\circ}$ e $3^{\circ}$. In MATTOS, L.P. (Org.), Estatuto da cidade comentado: Lei $\mathrm{n}^{\circ}$ 10.257, de 10 de julho de 2001, Belo horizonte: Mandamentos, 2002, pp. 79-106, p. 86.

SOUSA JÚNIOR, J.G.de. Direito como liberdade: o Direito achado na rua. Experiências populares emancipatórias de criação do Direito, Tese de Doutorado, Universidade de Brasília, 2008.

, Conferencia - Aula Magna: Direito achado na rua. Disponible en: http://www.youtube.com/watch?v=fw2eIsZJXZ8. Acesso em 24/05/2010.

SOUSA SANTOS, B., Critica de la razón indolente: contra el desperdício de la experiência, Bilbao, Ed. Desclée de Brouwer, 2000.

TSIOMIS, Y. O ensino do projeto urbano entre a crise e a mutação. In: MACHADO, D.B.P. (Org.). Sobre Urbanismo. Rio de Janeiro: Viana\&Mosley; Prourb, 2006

WARAT, L.A. Mitos e teorias na interpretação da lei. Porto Alegre, Síntese, 1979. O abuso estatal do Direito. Revista Sequência, núm. 21, vol. 11, 1990, pp. 34-50.

WOLKMER, A.C, Pluralismo Jurídico. Fundamentos de uma nova cultura no Direito, São Paulo, Alfa-omega, 2001.

Entrevista para Universidad Andina Simón dentro de la VIII Conferencia Latinoamericana de Crítica Jurídica, Ecuador, 30 de septiembre al 2 de octubre de 2013. 